\title{
BMJ Open How and why do women's groups (WGs) improve the quality of maternal and child health (MCH) care? A systematic review of the literature
}

\author{
Karla Canuto, ${ }^{1,2}$ Robyn Preston (D) , ${ }^{3,4}$ Sam Rannard, ${ }^{5}$ Catrina Felton-Busch,, 6 \\ Lynore Geia, ${ }^{8,9}$ Lee Yeomans, ${ }^{10}$ Nalita Turner, ${ }^{4,11}$ Quitaysha Thompson, ${ }^{12,13}$ \\ Karen Carlisle (D) , ${ }^{4}$ Rebecca Evans, ${ }^{4}$ Megan Passey, ${ }^{14}$ Sarah Larkins (D) , ${ }^{4}$ \\ Michelle Redman-MacLaren (D) , ${ }^{15}$ Jane Farmer (D) , ${ }^{16}$ Melody Muscat, ${ }^{4,17}$ \\ Judy Taylor ${ }^{4}$
}

To cite: Canuto K, Preston R, Rannard S, et al. How and why do women's groups (WGs) improve the quality of maternal and child health (MCH) care? A systematic review of the literature. BMJ Open 2022;12:e055756. doi:10.1136/ bmjopen-2021-055756

- Prepublication history and additional supplemental material for this paper are available online. To view these files, please visit the journal online (http://dx.doi.org/10.1136/ bmjopen-2021-055756)

Received 22 July 2021 Accepted 31 January 2022

Check for updates

(C) Author(s) (or their employer(s)) 2022. Re-use permitted under CC BY-NC. No commercial re-use. See rights and permissions. Published by BMJ.

For numbered affiliations see end of article.

Correspondence to

Robyn Preston;

r.preston@cqu.edu.au

\section{ABSTRACT}

Background This systematic review was undertaken to assist the implementation of the WOmen's action for Mums and Bubs (WOMB) project which explores Aboriginal and Torres Strait Islander community women's group (WG) action to improve maternal and child health (MCH) outcomes. There is now considerable international evidence that WGs improve MCH outcomes, and we were interested in understanding how and why this occurs. The following questions guided the review: (1) What are the characteristics, contextual influences and group processes associated with the MCH outcomes of WGs? (2) What are the theoretical and conceptual approaches to WGs? (3) What are the implications likely to inform Aboriginal and Torres Strait Islander WGs?

Methods We systematically searched electronic databases (MEDLINE (Ovid); CINAHL (Ebsco); Informit health suite, Scopus, Emcare (Ovid) and the Cochrane Library and Informit), online search registers and grey literature using the terms mother, child, group, participatory and community and their variations during all time periods to January 2021. The inclusion criteria were: (1) Population: studies involving community WGs in any country. (2) Intervention: a program/intervention involving any aspect of community WGs planning, acting, learning and reviewing $\mathrm{MCH}$ improvements. (3) Outcome: studies with WGs reported a component of: (i) $\mathrm{MCH}$ outcomes; or (ii) improvements in the quality of $\mathrm{MCH}$ care or (iii) improvements in socioemotional well-being of mothers and/or children. (4) Context: the primary focus of initiatives must be in community-based or primary health care settings. (5) Process: includes some description of the process of WGs or any factors influencing the process. (6) Language: English. (7) Study design: all types of quantitative and qualitative study designs involving primary research and data collection.

Data were extracted under 14 headings and a narrative synthesis identified group characteristics and analysed the conceptual approach to community participation, the use of theory and group processes. An Australian typology of community participation, concepts from Aboriginal and Torres Strait Islander group work and an adapted

\section{Strengths and limitations of this study}

- Our systematic review was methodologically robust, adhering to the Preferred Reporting Items for Systematic Reviews and Meta-analyses protocol and with a prepublished protocol and registration on PROSPERO.

- Multiple authors, both Aboriginal and Torres Strait Islander and non-Indigenous, were involved in each step of the review, increasing the rigour of our analysis

- International review including documents from both high-income and low-income countries.

- Limitations include difficulty with defining search terms that would ensure incorporation of all studies that used women's groups for maternal and child health improvement and the diversity of material retrieved in terms of amount of detail.

- Furthermore, there are concerns about the methodological soundness of some of the included documents and studies, and appropriate quality assessment particularly where multiple methods are used.

framework of Cohen and Uphoff were used to synthesise results. Risk of bias was assessed using Joanna Briggs Institute Critical Appraisal Tools.

Results Thirty-five (35) documents were included with studies conducted in 19 countries. Fifteen WGs used participatory learning and action cycles and the remainder used cultural learning, community development or group health education. Group activities, structure and who facilitated groups was usually identified. Intergroup relationships and decision-making were less often described as were important concepts from an Aboriginal or Torres Strait Islander perspective (the primacy of culture, relationships and respect). All but two documents used an explicit theoretical approach. Using the typology of community participation, WGs were identified as predominantly developmental (22), instrumental (10), empowerment (2) and one was unclear. 
Discussion A framework to categorise links between contextual factors operating at micro, meso and macro levels, group processes and $\mathrm{MCH}$ improvements is required. Currently, despite a wealth of information about WGs, it was difficult to determine the methods through which they achieved their outcomes. This review adds to existing systematic reviews about the functioning of WGs in $\mathrm{MCH}$ improvement in that it covers WGs in both high-income and low-income settings, identifies the theory underpinning the WGs and classifies the conceptual approach to participation. It also introduces an Australian Indigenous perspective into analysis of WGs used to improve MCH.

PROSPERO registration number CRD42019126533.

\section{INTRODUCTION}

In the last 30 years, community participation interventions with women's groups (WGs) using participatory learning and action (PLA) cycles as an intervention to improve maternal and child health $(\mathrm{MCH})$ have flourished, especially in low-income countries. ${ }^{12}$ Other types of WGs designed to lead to $\mathrm{MCH}$ improvement have also proliferated. In this paper, we use WGs to refer to all groups, whether or not they use PLA approaches.

WGs are generally understood in the international $\mathrm{MCH}$ literature as community-based organised groups of women who are using PLA cycles, health promotion techniques, peer support, two-way learning or community development, to mobilise individual and/or community action for health or social outcomes. ${ }^{3}$ They are used to involve local women in identifying needs and priorities for $\mathrm{MCH}$ improvement as well as increasing reach of primary healthcare (PHC), and providing new information or services.

The word intervention carries a dual meaning. As a research team, the understanding of WGs as an intervention is in accordance with the WHO definition of a health intervention:

A health intervention is an act performed for, with or on behalf of a person or population whose purpose is to assess, improve, maintain, promote or modify health, functioning or health conditions. ${ }^{4}$

On the other hand, the use of the word intervention in the Aboriginal and Torres Strait Islander health context carries negative implications of a sociopolitical act of government enacted on Aboriginal and Torres Strait Islander peoples and raises conflicting understandings and emotions for many. The term carries emotionally weighted meanings of an ongoing hegemonic deficit narrative of 'problematising' Aboriginal and Torres Strait Islander peoples. This creates a pall of stigmatisation of communities as places of darkness and violence, and diminishes the inclusion of perspectives of community self-determination and their agency in addressing problematic issues and seeking solutions to community identified issues. ${ }^{5}$ As an Indigenous and non-Indigenous research team and authors, we stress the importance of reflecting on the deeper meaning of the word intervention when used in Aboriginal and Torres Strait Islander health. In Aboriginal and Torres Strait Islander health, the term initiative is preferred over intervention.

A systematic review and meta-analysis of the outcomes of groups conducted in Nepal, Malawi, Bangladesh and India found improved $\mathrm{MCH}$ where there is adequate population coverage and an adequate proportion of pregnant women participate. ${ }^{3}$ The Strong Women, Strong Babies, Strong Culture programme in Australian Aboriginal communities is embedded in Aboriginal culture and uses a community development approach with WGs to bring about improvements to $\mathrm{MCH}^{6}$

While the results of the WGs in rural, low-income countries in improving $\mathrm{MCH}$ indicators appear clear, the group processes through which they exert their influence are less clear. If WGs are to be applied more generally, then analysis is important to identify facilitation styles, leadership, cultural influences and relationships that might best bring about positive outcomes. ${ }^{78}$ In Australia, in groups involving Aboriginal and Torres Strait Islander women, the wisdom and practical knowledge of Elders is foundational as well as knowledge of the cultural context, community history and the nature of relationships. ${ }^{9}$

The challenges in conducting primary research about WGs are accepted. There are numerous variables that may affect functioning including whether groups are newly constructed or existing, the nature and extent of community participation, the type of facilitation and the alignment with the country's health system. In addition, the extent to which contextual factors such as community norms and culture affect the operation of the WGs is important. Further research might consider these variables more systematically in order to uncover aspects that might be associated with MCH improvements. ${ }^{18}$

In Australia, the setting for this review, Aboriginal and Torres Strait Islander peoples has been working for decades to address the health disparities they experience. ${ }^{10}$ The establishment of Aboriginal and Torres Strait Islander community controlled, culturally responsive and comprehensive primary healthcare services in over 150 locations in Australia has been a life-changing achievement for the Aboriginal and Torres Strait Islander community. ${ }^{11}$ The research project, WOmen's action for Mums and Bubs (WoMB), was designed to explore the involvement of community women in identifying and implementing strategies to improve $\mathrm{MCH}$ outcomes in Aboriginal and Torres Strait Islander communities. A non-randomised step wedge implementation trial was designed using WGs as an initiative. ${ }^{12}$ Ideally, WGs are community driven and self-determining; consistent with the principles of Aboriginal and Torres Strait Islander women making decisions about the issues they experience with MCH care and the type of healthcare they want; healthcare that respects culture and relationships. ${ }^{13}$

In order to inform the WoMB study, we conducted a systematic review to synthesise and critically analyse the currently available evidence on the characteristics, approaches to community participation, use of theory and group processes associated with the $\mathrm{MCH}$ outcomes 


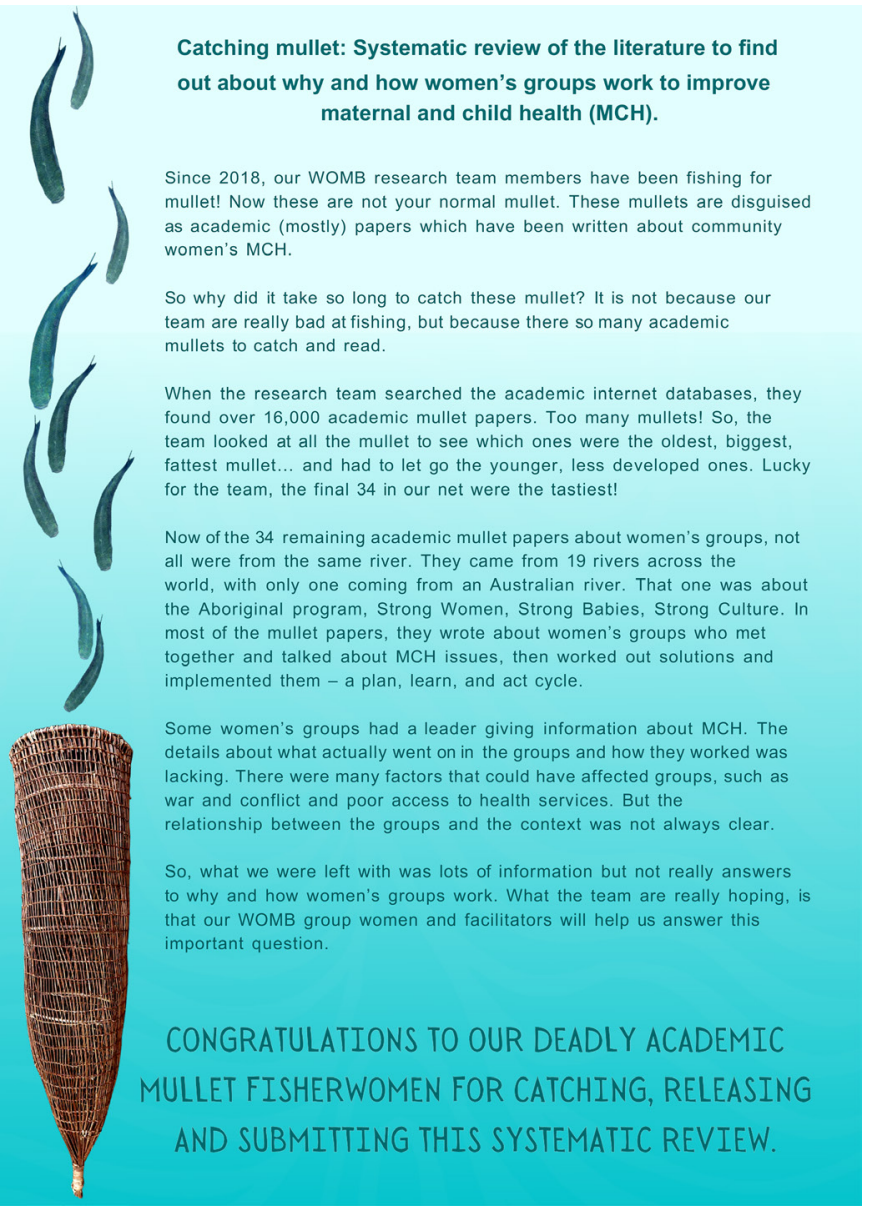

Figure 1 Catching mullet: community explanation of this systematic review.

of WGs. To meet this aim, the following questions guided the review:

1. What are the characteristics, contextual influences and group processes that are associated with the MCH outcomes of WGs?

2. What are the theoretical and conceptual approaches to WGs?
3. What are the implications likely to inform Aboriginal and Torres Strait Islander WGs?

\section{METHODOLOGY}

The review was conducted between July 2018 and January 2021 by a multidisciplinary team including Aboriginal and Torres Strait Islander and non-Indigenous researchers and practitioners involved in the WoMB project. A published protocol described methods and followed the definition of 'systematic review' in the Preferred Reporting Items for Systematic Reviews and Meta-analyses for Protocols (PRISMA-P) guidelines. ${ }^{141516}$

\section{Patient and public involvement}

Members of WoMB groups were involved in the reporting and dissemination plans of the systematic review. Catching Mullet (figure 1) provides a community explanation of this systematic review.

\section{Inclusion criteria}

The inclusion criteria are outlined in table 1. For this review, community-based WGs are included if they involve any aspect of planning, acting, learning or reviewing MCH improvements. Peer-reviewed and grey literature from all time periods was included. Non-peer reviewed literature was also searched given most WG programmes are in low-resource settings or implemented outside of academic institutions (eg, by non-government organisations, NGOs).

\section{Information sources}

A systematic search was conducted in: MEDLINE (Ovid); CINAHL (Ebsco); Informit health suite, Scopus, Emcare (Ovid) and the Cochrane Library to 19 August 2019. An updated search of these databases was conducted in January 2021 excluding Informit as the search function had changed.

The selection of groups to be included in grey literature searching was based on the researchers' professional

Table 1 Inclusion criteria

\begin{tabular}{|c|c|}
\hline $\begin{array}{l}\text { Criteria by PICO } \\
\text { headings }\end{array}$ & Inclusion \\
\hline Population & Studies involving community WGs in any country. \\
\hline Outcome & $\begin{array}{l}\text { Studies with WGs reported a component of: (i) MCH outcomes; or (ii) improvements in the quality of } \\
\mathrm{MCH} \text { care or (iii) improvements in socioemotional well-being of mothers and/or children. }\end{array}$ \\
\hline Process & Includes some description of the process of WGs or any factors influencing the process. \\
\hline Time period & All time periods. \\
\hline Language & English. \\
\hline Study design & All types of quantitative and qualitative study designs involving primary research and data collection. \\
\hline
\end{tabular}

$\mathrm{MCH}$, maternal and child health; WGs, women's groups. 
Table 2 Search terms

\section{Mothers and children's groups}

\begin{tabular}{|c|c|c|c|c|}
\hline Mother & Child & Group & Participatory & Community \\
\hline $\begin{array}{l}\text { mother* mom* } \\
\text { mum women } \\
\text { woman maternal }\end{array}$ & $\begin{array}{l}\text { child* infant }^{*} \text { baby babies } \\
\text { bub antenatal prenatal birth* } \\
\text { play* neonat* newborn }^{*} \\
\text { pregnan* postnatal }\end{array}$ & $\begin{array}{l}\text { group }{ }^{\star} \text { circle }^{\star} \text { class }^{*} \\
\text { club }^{*} \text { committee }^{*} \\
\text { meeting }^{\star} \text { program }^{\star} \\
\text { facilitat* }^{*}\end{array}$ & $\begin{array}{l}\text { participat* involve* } \\
\text { empower* engag* }\end{array}$ & $\begin{array}{l}\text { network* stakeholder* } \\
\text { peer* } \\
\text { 'self-help' }\end{array}$ \\
\hline
\end{tabular}

experience in the field and knowledge of key organisations within this space. Two reviewers (RP and JT) contacted authors research groups in Australia and internationally; and WGs by email to obtain grey literature that covered processes or mode of working of community-based WGs. Hand-searching, the reviewing of hard copy papers from journals or departments that were not available on databases at the time, was conducted. No new content was included in the final review from these inquiries.

\section{Search terms}

Search terms outlined in table 2 relate to population, and context. Full-search strategies are available in online supplemental table 1. Outcomes (health and other) were incorporated in data extraction. All populations were included.

\section{Other data sources}

Grey literature sources were identified in October 2019 using native site search interfaces on key organisational websites (eg, WHO, USAID, HealthInfoNet and others) and Google searches restricted to the key organisations' domains (table 3). Individual authors (RP, JT, SL, RE, MP, LY, KCar, KCan and MR-M) scanned for relevant documents, with the first 100 results being checked for each search. If results were still relevant at 100 , authors continued reviewing until documents were no longer relevant.

\section{Study selection}

One author (JT) conducted a first screen of title and abstract of all documents. Another author (RP) reviewed 100 in every 1000 documents. Discrepancies were reviewed by a third reviewer (RE or SL). All unique documents obtained through our search of databases, websites, reports, and other sources were imported into Colandr for screening. ${ }^{17}$

All documents that met the inclusion criteria from title and abstract review or those that could not be excluded had the full text retrieved. Full texts were independently reviewed by two team members (SL and RE). Any discrepancies for inclusion were resolved by arbitration by a third team member (MP). Reference lists of included documents were also reviewed for relevant inclusions.

Table 3 Grey literature

\begin{tabular}{|c|c|c|}
\hline Organisation & Source & Search string \\
\hline WHO https://apps.who.int/iris/discover? & WHO IRIS & $\begin{array}{l}\text { (women OR mothers) AND (group } \\
\text { OR groups) AND participatory }\end{array}$ \\
\hline WHO site: who.int & \multirow{9}{*}{$\begin{array}{l}\text { Google Scholar: } \\
\text { site search }\end{array}$} & \multirow{9}{*}{$\begin{array}{l}\text { ('womens groups' OR ‘women's } \\
\text { groups' OR 'women's group' } \\
\text { OR 'mothers group' OR } \\
\text { 'mothers groups' OR 'women's } \\
\text { participatory groups') AND } \\
\text { participatory AND (infant OR } \\
\text { child) }\end{array}$} \\
\hline United Nations Children's Fund (UNICEF) site: unicef.org & & \\
\hline Oxfam site: oxfam.org & & \\
\hline $\begin{array}{l}\text { Australian Department of Foreign Affairs and Trade (DFAT) site: dfat. } \\
\text { gov.au }\end{array}$ & & \\
\hline $\begin{array}{l}\text { GIZ (Deutsche Gesellschaft fur Internationale Zusammenarbeit } \\
\text { GmbH) site: giz.de } \\
\text { International Women's Development Agency (IWDA) site: iwda.org.au }\end{array}$ & & \\
\hline Save the Children UK site: savethechildren.org.uk & & \\
\hline Save the Children Australia site: savethechildren.org.au & & \\
\hline $\begin{array}{l}\text { United States Agency for International Development (USAID) site: } \\
\text { usaid.gov }\end{array}$ & & \\
\hline World Bank site: worldbank.org & & \\
\hline
\end{tabular}




\section{Data extraction}

Full-text documents were downloaded from Colandr and two reviewers (KCar and JT) extracted information onto a data collection sheet, for each document, covering country, location, cultural lens of researchers, study design, theoretical base, conceptual approach to participation, group length of operation, reason and initiator, membership, decision-making, intragroup relationships and cultural, community, political and institutional contextual factors, study quality and $\mathrm{MCH}$ and socioemotional well-being outcomes. Approximately 20\% of each reviewer's data extraction were checked by a third reviewer (RP) for consistency.

Data were summarised and transferred into two tables (JT, RE and KCar). Online supplemental table 2 included group characteristics, conceptual approach to community participation and theoretical underpinning. Online supplemental table 3 included material about group process including contextual factors influencing group process. Any discrepancies were resolved through consensus-based discussion or a fourth reviewer (SL).

\section{Quality assessment of studies}

The Joanna Briggs Institute Critical Appraisal Tools (JBI Tools) for assessing quantitative and qualitative studies were used to assess the quality of included documents, including risk of bias. ${ }^{18}$ Documents using multimethods were assessed against relevant approaches. Three authors (KCar, RP and JT) undertook an initial quality review using the JBI tools. One author (SL) then reviewed any discrepancies and categorised/scored the documents based on the tool checklists: low quality (below 5), moderate (6-7) and high $(8+)$. No documents were excluded on quality grounds (online supplemental table 4).

\section{Data synthesis}

Qualitative comparative analysis and narrative synthesis was conducted from the extraction tables under three headings; group characteristics, conceptual approach to participation and use of theory and group processes, by a team of researchers (JT, RE, KCar, LY, KCan, MP and JF). Well-recognised frameworks for examining the conceptual approach to participation were examined. ${ }^{1920}$ However, we used an Australian community participation typology derived from research with rural communities, NGOs and health professionals working to plan, identify needs and implement action to improve health. ${ }^{21}$ This review was conducted to inform a trial involving an initiative with WGs within Aboriginal and Torres Strait Islander communities-thus our group of investigators felt that the communitarian and collectivist focus was important.

A further review by Aboriginal and Torres Strait Islander team members (KCan, LY, NT, CF-B, QT, YCJ and LG) considered concepts for WGs in Aboriginal and Torres Strait Islander WGs, which includes the primacy of culture, relationships and respect. ${ }^{13}$
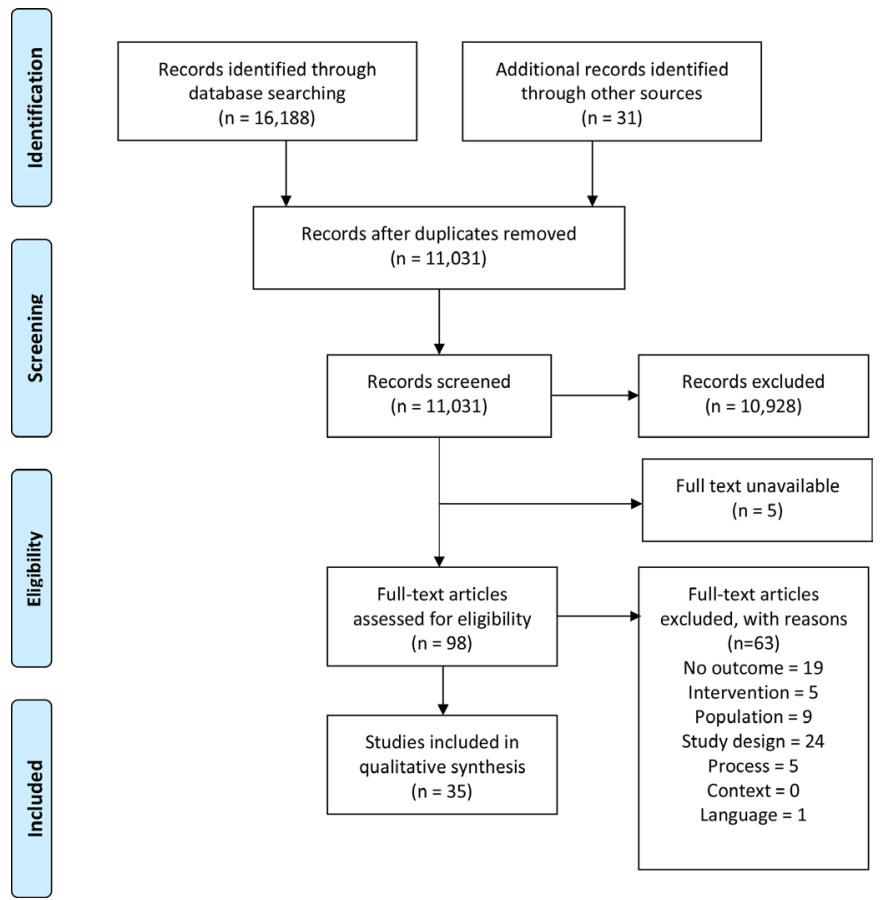

Figure 2 PRISMA flowchart of document inclusion. ${ }^{70}$ PRISMA, Preferred Reporting Items for Systematic Reviews and Meta-analyses.

\section{RESULTS}

The database and grey literature searched between August 2019 and January 2021 identified 11031 unique documents. After initial screening by title, and abstract, 98 papers were included for full-text screening, 35 met the inclusion criteria and have been included in this review (figure 2). Details of the 63 full texts that were excluded are available in online supplemental table 5.

\section{The scope of the studies}

The 35 included documents are described in online supplemental tables 2 and 3. The documents were published between 1985 and 2020 about WGs in 19 countries. Twenty-five were conducted in low-income and lowmiddle-income countries using the 2019 World Bank Indicators. ${ }^{22}$ Ten were conducted in middle-income and high-income countries including one in Australia with Aboriginal women. ${ }^{6}$ In the middle-income and highincome countries, almost all groups were conducted in economically poor communities, some with limited access to MCH services. The primary stated goal for WG activity, reported in all documents, was to improve $\mathrm{MCH}$ or women's socioemotional outcomes, increase information about MCH for women or assist in accessing care.

Study designs included 13 qualitative studies (evaluations and descriptive studies), 10 mixed-method evaluations, four quasi-experimental designs, six randomised controlled trials and two quantitative cross-sectional studies. The quality of the studies varied, with nine studies classified as high, 16 as moderate and 10 as low quality (online supplemental table 4). 
What are the characteristics, mechanisms and contextual influences associated with the outcomes and effectiveness of WGs?

\section{Group characteristics}

The majority of WGs reported on were initiated by NGOs including international aid agencies, ${ }^{23-33}$ researchfocussed NGOs ${ }^{2}{ }^{31-41}$ or research teams. ${ }^{42-46}$ In eight studies the country's government ${ }^{647-53}$ was responsible for initiating WGs. Information was unavailable for two documents. ${ }^{54}$ Twenty-two studies reported WGs that were new or remobilised ${ }^{2} 623-2527-34363940444550525455$ and the remainder used existing groups. ${ }^{35} 38414648495153$ All groups included MCH education, two-way learning, awareness raising or information sharing components. Identifying community needs, issues and solutions was a stated activity in 23 groups. A broad range of other activities were covered by WGs.

\section{Group processes}

The reporting framework used for the presentation of results is that of Cohen and Uphoff ${ }^{56}$ as it includes wellrecognised group process concepts; group membership, group structure, leadership/facilitation, group inter-relationships, cultural/contextual factors affecting process, and decision-making.

\section{Group membership}

All groups were comprised local community women. Four documents reported that although primarily aimed at women, some groups also included some men, ${ }^{45} 51$ either as expectant fathers ${ }^{44}$ or the entire household. ${ }^{52}$ In four groups ${ }^{43-46}$ membership was discussed with village elders some of whom made referrals.

\section{Group structure and leadership/facilitation}

The 15 WGs that used a PLA cycle or other methods of identification of local issues, potential solutions and actions were led by local trained facilitators. ${ }^{263133-3739-434654}$ The four documents reporting on a community development approach involving autonomous programming and independent decision-making were also usually led by local trained facilitators. ${ }^{27445} 49$ Those WGs primarily providing health promotion/education delivered in a group setting were usually led by a health professional. ${ }^{23} 242838474851-5355$ These groups appeared to involve a didactic educative programme on aspects of MCH. Sometimes education was provided in a group context for reasons of efficiency rather than to benefit from group processes. Three documents used a collective problem solving approach, with peer support and new learning and shared leadership. ${ }^{29} 30$ Two documents reported on sharing cultural learning with women and health professionals usually without a set programme. Facilitation in these groups involved local women knowledgeable about some aspects of culture. ${ }^{628}$

\section{Decision-making}

Thirteen documents reported that decision-making was enacted by WG members 252731333436384145495153 or through the combined efforts of WG members, group facilitators, research teams and the wider community. ${ }^{26}$ 28-30 39424654 In one document, decision-making was at a level removed from the WG that is, sitting with a research team. ${ }^{24}$ For a proportion of documents, it was unclear where decision-making rested. 6233237434748505255

\section{Community/cultural contextual factors affecting group processes}

Twenty-six documents provided a rich description of community and cultural factors in the background to the study. In 19 documents, contextual factors were linked to group processes although usually this was a passing reference rather than an analysis. Linked factors included gender imbalance affecting women's decision-making and leadership, ${ }^{2636454649}$ the need to take account of different castes, tribes and languages in group makeup, ${ }^{37} 39404346$ and the presence of deep-rooted cultural and spiritual beliefs that might limit MCH. ${ }^{2} 32354465455$ In considering cultural factors affecting WGs, there was some nuanced discussion. Morrison et $a l^{2}$ reported on the cultural phenomenon of 'ke garne', a feeling that one has no personal control over one's life circumstances, that might have impacted on women's ability to believe that they could make improvements. ${ }^{2}$ A study conducted in Orange County Florida ${ }^{29}$ reported that the WGs struggled with envisioning change and discussions often did not move beyond the immediate needs of the participants. There were reports of the presence of valuable cultural factors that enhance MCH. ${ }^{625}$ Lowell et at reported on the positive influence Aboriginal cultural knowledge about birthing can have in adding to mainstream health knowledge and practice.

Seventeen documents described aspects of the sociopolitical context in Nepal, Sri Lanka, Myanmar and India that may have affected WGs. Eight mentioned war or political instability which restricted movement, access to health services and created food insecurity and promoted the emergence of female or child-headed households. ${ }^{31} 363941$ 47-49 53 A study conducted in Myanmar ${ }^{47}$ described political instability, climate change and armed conflict in Kayah State that affected group functioning.

\section{Reflexivity}

There was rarely a mention of the impact of the researchers' location, position or culture on their interpretation of aspects of the WGs' functioning. This applied equally to researchers located within or outside the country. The exception was Morrison et al who acknowledged several aspects of Nepal's culture that might have impacted on group processes and the non-Nepali's facilitation role. ${ }^{54}$

\section{The use of theory and the conceptual approach to WG participation}

Use of theory

All documents, apart from two ${ }^{52} 53$ provided an explicit theoretical approach to WGs. There were a wide range of theories used with 29 using theory about community participation/mobilisation, capacity-building, 
Table 4 Conceptual approaches to community participation in WGs in health improvement

$\begin{array}{ll}\begin{array}{l}\text { Contributions } \\ \text { approach }\end{array} & \begin{array}{l}\text { To gain resources, time, labour or expertise } \\ \text { from the women or community } \\ \text { The women have no say in the direction of the } \\ \text { project }\end{array} \\ \begin{array}{l}\text { Instrumental } \\ \text { approach }\end{array} & \begin{array}{l}\text { Pre-established goals and outcomes } \\ \text { Driven by a leader (usually a health }\end{array} \\ \text { professional) } & \text { Uses a set programme } \\ \text { Empowerment } & \text { Women have a choice to change aspects of } \\ \text { approach } & \text { their lives } \\ & \text { Encourages women's control of these aspects } \\ & \text { Encourages women to take steps towards } \\ \text { change }\end{array}$

WGs, women's groups.

development or empowerment. Nine used the theories/ concepts of Alinsky, ${ }^{27}$ Freire ${ }^{29} 3946$ and Putnam, ${ }^{27}$ or theories of healthcare production and demand, ${ }^{37}$ autodiagnosis, ${ }^{26}$ gender equity ${ }^{51}$ Bandura's social learning and social cognitive theory ${ }^{54}$ and two-way learning. ${ }^{6}$ A further four documents used theory of agency, ${ }^{35}$ the UK Deptford model ${ }^{50}$ health belief model ${ }^{25}$ or a social work practice framework including feminist practice. ${ }^{30}$

\section{Conceptual approach to WG participation}

A typology of the conceptual approach to community participation (table 4) was used to identify conceptual approaches used in the WGs and align these, if possible, with aspects of group process. ${ }^{21}$ In most documents, there was evidence of more than one approach, but we classified the group by consensus according to the predominant one (RP, KCar and JT). Twenty-two used predominantly a developmental approach, reflecting an interactive evolutionary process in the groups. ${ }^{2} 6252730-3739-464954$ Ten documents reported on groups using a predominantly instrumental approach with predetermined goals and outcomes and a set structure. . $^{232438474851-5355}$ Two used a predominantly empowerment approach with a focus on enabling women to make decisions, ${ }^{46}{ }^{50}$ with one unable to be classified based on the available information. ${ }^{26}$

There was alignment between the overall conceptual approach to community participation and group structure. All of the groups using PLA cycles, except one where we could not classify the conceptual approach, used a developmental approach. In addition to the groups using PLA, those four studies using community development enabling the women to develop autonomous programming and independent decision-making, were also all developmental. ${ }^{27} 444549$ The studies reporting using a collective problem-solving approach, with peer support and new learning were also developmental. Two of these used a developmental, ${ }^{30}$ and one an empowerment approach. ${ }^{29}$ Two documents reported on sharing cultural learning with women and health professionals and used a developmental approach. ${ }^{628}$ The 10 groups reported in studies that used didactic health promotion in a group setting were all classified as having an instrumental approach. ${ }^{23} 242838474851-5355$

\section{The results likely to inform Aboriginal and Torres Strait Islander WGs}

The following concepts, from an Aboriginal and Torres Strait Islander lens, were used to examine the studies: the primacy of culture, relationships and respect. ${ }^{13}$ Withingroup relationships were one aspect of reporting that had some relevance to Aboriginal and Torres Strait Islander WGs. Just over half of the documents reported on group members supporting each other. From an Aboriginal and Torres Strait Islander perspective, within-groups relationships would be considered fundamental and likely influence all aspects of WGs developed for $\mathrm{MCH}$ purposes. In two documents, ${ }^{6}{ }^{25}$ sharing cultural activities and knowledge was described. One of these was reporting the value of Australian Aboriginal knowledge ${ }^{6}$ and practice in $\mathrm{MCH}$ and another promoting women's use of Ecuadorian Quichua traditional foods to improve nutrition. ${ }^{25}$

\section{DISCUSSION}

What are the characteristics, contextual influences and mechanisms that are associated with the outcomes and effectiveness of WGs?

The strength of this review is that a wealth of information was compiled from the studies describing the characteristics of WGs, their establishment and the operation of community, cultural, institutional and political contextual factors. The information comes from diverse contexts in different countries, with differently structured groups and alignment to state/country health systems. What was common was an overall aim to progress $\mathrm{MCH}$ and/or social well-being through WGs.

To understand how the WGs achieved their health or well-being outcomes from the data available was challenging. This is not a new finding, and is consistent with Byass's commentary about the complexity of achieving a link between specific real-life inputs such as WGs and health improvements, and the focus on outcomes rather than the processes that might achieve them. ${ }^{57}$ Abimbola suggests that achieving a broad understanding of community participation processes should be a 'holy grail' and he contributes an in-depth study about the group facilitator's role in WGs. ${ }^{58}$ More of this type of analysis would be useful in establishing the key factors relevant to successful WGs.

The use of WGs to assist women to develop knowledge, plan and make decisions about $\mathrm{MCH}$ improvements requires that the groups involve active participation. There is extensive recent commentary on the importance of participation in aspects of health improvement and providing care of high value to participants 
including MCH. ${ }^{138-65}$ While health policy-makers adhere to this maxim, the difficulty lies in how 'participatory' components of health system activities should become. Furthermore, the processes to effectively implement this participation should draw on available evidence. The usual role of didactic health information giving must be adjusted if participants are to be actively involved. ${ }^{50}$

In the 22 documents classified as developmental, there are references to WG members making decisions necessitating interaction. However, facilitators in these groups would have had to overcome perceptions that receiving health information is a one-way didactic process. In the 10 instrumental WGs, the extent of interaction and decision-making was difficult to determine; for example, whether women's suggestions could influence the topics to be addressed.

A step towards achieving a better understanding of how WGs might improve MCH could be the development of a theoretical frame or set of variables that could be used to examine a WG across a life course or timeline. If variables, such as we used in analysing studies about WGs structure and processes, were consistently applied when evaluating WGs, then a conceptual model of the interaction between different aspects of groups could be developed. Morrison et al began this process as they summarised their findings regarding processes through which WGs led to equitable behaviour change including learning and developing knowledge, social support gained through group participation and the process of taking action. ${ }^{54}$

The reporting of contextual factors affecting group structure and process remains difficult. Again, a framework to consider community, cultural, institutional and political factors at the micro, meso and macro levels would prove helpful. In these studies, macro (national or state) political factors such as political upheaval and security issues were well documented and the impact clear. ${ }^{36} 41$ Grouping variables at the meso (community) and micro (WG) levels such as traditional attitudes towards birthing (meso) and membership of WGs being advised by male elders (micro) might clarify the link to group functioning.

Regarding the importance of culture, there were documents in which the integration of culture into WGs was strong. For example, traditional Quichua values about participating for the common good and the nutritional value of consuming certain greens (meso level factors) influenced the way WGs were organised in one study. ${ }^{25}$

Systematic reviews show that WGs have been applied with the goal of improved $\mathrm{MCH}$ and well-being in countries with all income levels. ${ }^{63}$ From these reviews, it is the improvements that have occurred in low-income and lowmiddle income settings that appear to justify the scaling up and extension of WGs where facilitators can be drawn from a cadre of local workers/volunteers. ${ }^{38415766}$ In highincome settings, the situation is different regarding the role of volunteers in usually strong health systems.

To effectively scale up WGs in countries of all income levels, better understanding of the role of facilitation, ${ }^{58}$ cultural influences ${ }^{1367}$ and the group's structural arrangements ${ }^{6}$ is key. Without this understanding, transferring an initiative to other countries and cultures might amount to transposing programmes from very different contexts and cultures with minimal adaptation. ${ }^{68}$

Understanding the sustainability of a community participation initiative beyond a project initiative is also important. Sondaal et al sought to investigate the sustainability of the WGs in Makwanpur established by the NGO Mother and Infant Research Activities, once the research was completed. ${ }^{69}$ These authors found that over $80 \%$ of the groups continued, especially if they were considered important at the local level and new information was being provided.

\section{What are the theoretical and conceptual approaches to WGs?}

In a systematic review of rural community participation in PHC, Preston et al found limited use of theory (there was not a 'theory' data extraction category) to support initiatives and some conceptual confusion in approaches to community participation. ${ }^{62}$ In this review, 10 years later, the predominant conceptual approach to community participation was identifiable, except in one document. Almost all documents referenced a theoretical basis, although there was a wide range of theories and different levels of description. Usually theory about community participation/engagement or capacity building was used and there was evidence of developing theoretical frameworks about WGs, such as Morrison's et als study described above..$^{54}$ The value in theory-driven initiatives, is that theory might provide a foundation for consistent evidence gathering about process and outcomes, which in turn might inform the effectiveness of future initiatives.

WG's outcomes have been measured through randomised controlled trials, mixed-method studies and descriptive qualitative research. Mannell and Davis suggest that qualitative methods are necessary to fully understand interventions and their effects. ${ }^{64}$ Currently, innovative qualitative methods are being included as part of randomised controlled trials in intervention evaluation, realist evaluation and pragmatic trials in order to answer some of the questions about the processes involved in effecting health improvements.

In the future, it is important that there is more theoretical work that accounts for some of the messiness and uncertainty of community participation initiatives. Implementing community participation necessarily engages researchers in understanding complexity as an empirical reality and requires flexibility in approach. ${ }^{65}$ Theorising women's participation and action through group work, accommodating this messiness, might assist effective implementation.

\section{Implications for Aboriginal and Torres Strait Islander WGs}

As mentioned, this review was performed to inform an ongoing project implementing WGs to strengthen MCH with Aboriginal and Torres Strait Islander women in 10 locations in remote and rural Australia. ${ }^{12}$ Yet only one 
study reporting on the use of WGs in this setting was included in this review. Theory generation applicable to Australian Aboriginal and Torres Strait Islander WGs was difficult given that the studies reported on did not use the concepts of the primacy of culture, relationships, and respect in the same way.

Aboriginal and Torres Strait Islander authors reflected on this, concluding that the dearth of documents reporting on WGs in the Australian Indigenous context belies the long history of Aboriginal and Torres Strait Islander women's leadership in our everyday worlds and survival as a peoples, especially in the sphere of family well-being. Although much activity is occurring, little of this is reported in the academic literature, suggesting a need to get better at sharing our story using this medium! Nevertheless, the WoMB project will be informed through this systematic review, and we anticipate that the stories of facilitators and WGs will be told, and the theory will be elicited from the experiences of our women, facilitators and each of us. Using an Indigenist approach, our team proposes to use our new empirical data from Aboriginal and Torres Strait Islander women to generate more much needed theory and voice in a subsequent publication.

We also reiterate that researchers reflect on research language and use of terminology that is generally accepted in the research health field but may have negative meanings for Indigenous community partners and may impact on participatory processes. This means reflecting on the use of accepted health and research language that carry different meanings for people who have generational lived experience of government 'punitive intervention' policies and practices enacted on them. We suggest including strengths-based language and approaches when working with Aboriginal and Torres Strait Islander health reform processes.

\section{Limitations and strengths}

There were several factors about the methodology of this systematic review that may impact on the findings. These include the difficulty with defining search terms that would ensure incorporation of all studies that used WGs for MCH improvement; the diversity of material retrieved in terms of amount of detail, concerns about the methodological soundness of the included documents and studies and appropriate quality assessment particularly where multiple methods are used.

It was not always clear at the initial search whether or not a WG was participatory. In the first screen of documents, a judgement was made to include those where there was mention of a WG's involvement in any of the aspects of planning, identifying needs, decision-making or taking action. The level of participation described varied from women independently making decisions and running groups to women participants giving feedback about how they would use the information given in the groups. In including documents that met the criteria of women's involvement, we may have included reports of WGs that were not participatory.
The included documents varied significantly in the level of detail provided about the group, whether they were part of a programme or 'one-off' group, whether there were changes in the groups over time and whether they proceeded as planned.

The varying purposes of the studies reported may have accounted for the diverse methodological quality. Because of the need to capture as much information as possible about the functioning of WGs, a decision not to exclude studies of limited methodological quality was taken. Some of the early literature, for example Nishiuchi ${ }^{53}$ provided rich information but had limited description of methodology.

There were nine multimethod evaluations included in the studies and the quality assessment tools used did not include an adequate assessment for mixed-method studies. ${ }^{18}$ We completed a quality assessment for each of the components (quantitative and qualitative) and produced an overall composite score. This quality assessment did not consider the convergence or integration of data essential in mixed methodologies.

Despite this, our systematic review was methodologically robust, adhering to the PRISMA protocol and with a prepublished protocol and registration on PROSPERO ${ }^{15} 16$ Multiple authors, both Aboriginal and Torres Strait Islander and non-Indigenous were involved in each step of the review, increasing the rigour of our analysis.

\section{CONCLUSION}

Our systematic review adds to existing systematic reviews about the functioning of WGs in MCH improvement in that it covers WGs in both high-income and low-income settings, identifies the theory underpinning the WGs and classifies the conceptual approach to participation. It also introduces an Australian Indigenous perspective into analysis of WGs used to improve MCH.

This systematic review found that inconsistencies in the reporting of contextual and process issues made it difficult to determine the processes through which WGs achieved their health or well-being outcomes. We propose a framework adapted from that of Cohen and Uphoff ${ }^{56}$ of key concepts about group process that may assist in standardising the expectations for reporting. We also propose that contextual factors, including culture, affecting the WGs be discussed at the macro, meso and micro levels in order to assist establishment of links to group functioning. This might assist in addressing the methodological limitations identified in this review.

Only one study identified in this review involved Aboriginal and Torres Strait Islander women. ${ }^{6}$ This does not reflect a lack of activity of WGs in communities, rather a lack of reporting this activity through the peer-reviewed literature. As we learn from our current implementation of WGs to improve MCH with Aboriginal and Torres Strait Islander women, theory generation by the women in these groups may be advanced. 
Participation is a complex phenomenon; and most studies did not define the processes of participation. The exciting potential of participatory and codesign initiatives is reflected in widespread policy uptake, yet our nuanced and contextually informed understanding of how, in a practical sense, these groups are best introduced and supported still lags behind.

\section{Author affiliations}

${ }^{1}$ Aboriginal Health Equity, South Australian Health and Medical Research Institute, Adelaide, South Australia, Australia

${ }^{2}$ Naghir Tribe of the Kulkagul Clan, Torres Strait, Queensland, Australia

${ }^{3}$ School of Health, Medical and Applied Sciences, Central Queensland University,

Townsville, Queensland, Australia

${ }^{4}$ College of Medicine and Dentistry, James Cook University, Bebegu

Yumba,Townsville, Queensland, Australia

${ }^{5}$ Library and Information Services, James Cook University, Bebegu Yumba,

Townsville, Queensland, Australia

${ }^{6}$ Murtupuni Centre Rural and Remote Health, James Cook University, Mount Isa, Queensland, Australia

${ }^{7}$ Yangkaal and Gangaidda, Mount Isa, Queensland, Australia

${ }^{8}$ College of Healthcare Sciences, James Cook University, Bebegu Yumba, Townsville, Queensland, Australia

${ }^{9}$ Bwgcolman, Palm Island, Queensland, Australia

${ }^{10}$ Queensland Aboriginal and Islander Health Council, Brisbane, Queensland, Australia

${ }^{11}$ Anmatyerre/Jaru, Northern Territory, Northern Territory, Australia

${ }^{12}$ Gurindji Aboriginal Corporation, Kalkaringi, Northern Territory, Australia

${ }^{13}$ Gurindji woman, Kalkaringi, Northern Territory, Australia

${ }^{14}$ University Centre for Rural Health, University of Sydney, Lismore, New South Wales, Australia

${ }^{15}$ College of Medicine and Dentistry, James Cook University, Nguma-bada, Cairns, Queensland, Australia

${ }^{16}$ Swinburne Social Innovation Research Institute, Centre for Social Impact,

Swinburne University of Technology, Melbourne, Victoria, Australia

${ }^{17}$ Bijara, Charleville, Queensland, Australia

\section{Twitter Michelle Redman-MacLaren @shelmaclaren}

Acknowledgements We acknowledge WoMB facilitators, WoMB group members, Primary Health Care Staff and other WoMB researchers for their encouragement, support and feedback on this study. Professor Yvonne Cadet James (YCJ) provided guidance for the study design, conceptual advice and cultural knowledge. Talah Laurie designed figure 1. We thank Louise Armitage for proof-reading of the final document.

Contributors KCan: conceptualised and designed the study, contributed to the conception and design of this research, extracted data from individual studies, assisted with the analysis, critically reviewed the manuscript, provided information on methods design, contributed to the interpretation of the findings, revised the manuscript for important intellectual content, approved the final manuscript as submitted; SR: contributed to the conception and design of this research, performed the literature search and extracted data from individual studies, reviewed and revised the manuscript, provided information on data sources, methods design, revised the manuscript for important intellectual content, approved the final manuscript as submitted; CF-B, LG, NT and QT: reviewed and revised manuscript, gave cultural advice, contributed to interpretation of findings, approved the final manuscript as submitted; LY: contributed to the conception and design of this research, conducted the data analysis and interpreted the data, revised the first and subsequent drafts for important intellectual content, approved the final manuscript as submitted; KCar: contributed to conceptualisation and study design, contributed to methods design, extracted data from the included studies, contributed to data analysis and interpretation, assisted with draft revisions and critically reviewed the manuscript, approved the final manuscript as submitted; MP: contributed to conceptualisation and study design, assisted with data extraction, assisted with data integration and interpretation, critically reviewed the manuscript, approved the final manuscript as submitted; RE and SL: contributed to conceptualisation and study design, assisted in the literature search, screening and extracted data from individual studies, contributed to the interpretation of the findings, reviewed and revised the manuscript, revised the manuscript for important intellectual content, approved the final manuscript as submitted; MR-M: contributed to conceptualisation and study design, critically reviewed and revised the manuscript, contributed to the interpretation of the findings, revised the manuscript for important intellectual content, approved the final manuscript as submitted; JF: assisted with the analysis, reviewed and revised the manuscript, provided information on data sources, methods design, approved the final manuscript as submitted, MM: reviewed and revised manuscript; gave cultural advice, contributed to interpretation of findings, approved the final manuscript as submitted; RP and JT: conceptualised and designed the study, assisted with methods design, performed the literature search and extracted data from individual studies, conducted the data analysis and interpreted the data, wrote the first draft of the paper, revised the first and subsequent drafts for important intellectual content, approved the final manuscript as submitted. RP is guarantor and accepts full responsibility for the work and the conduct of the study, had access to the data, and controlled the decision to publish.

Funding Funded by the National Health and Medical Research Council (Australia): GNT1146013. This study is also part of the NHMRC funded CRE-Integrated Quality Improvement GNT1078927 (2015-2019) and CRE-STRIDE NHMRC GNT1170882 (2020-2025).

Competing interests None declared.

Patient consent for publication Not required.

Ethics approval This study does not involve human participants.

Provenance and peer review Not commissioned; externally peer reviewed.

Data availability statement No data are available. There is no data set as this is a systematic review.

Supplemental material This content has been supplied by the author(s). It has not been vetted by BMJ Publishing Group Limited (BMJ) and may not have been peer-reviewed. Any opinions or recommendations discussed are solely those of the author(s) and are not endorsed by BMJ. BMJ disclaims all liability and responsibility arising from any reliance placed on the content. Where the content includes any translated material, BMJ does not warrant the accuracy and reliability of the translations (including but not limited to local regulations, clinical guidelines, terminology, drug names and drug dosages), and is not responsible for any error and/or omissions arising from translation and adaptation or otherwise.

Open access This is an open access article distributed in accordance with the Creative Commons Attribution Non Commercial (CC BY-NC 4.0) license, which permits others to distribute, remix, adapt, build upon this work non-commercially, and license their derivative works on different terms, provided the original work is properly cited, appropriate credit is given, any changes made indicated, and the use is non-commercial. See: http://creativecommons.org/licenses/by-nc/4.0/.

Author note KCan and RP are joint first authors of this paper.

\section{ORCID iDs}

Robyn Preston http://orcid.org/0000-0003-4700-1521

Karen Carlisle http://orcid.org/0000-0002-1781-4057

Sarah Larkins http://orcid.org/0000-0002-7561-3202

Michelle Redman-MacLaren http://orcid.org/0000-0002-2055-7733

Jane Farmer http://orcid.org/0000-0003-1730-2622

\section{REFERENCES}

1 Farnsworth SK, Böse K, Fajobi O, et al. Community engagement to enhance child survival and early development in low- and middleincome countries: an evidence review. J Health Commun 2014;19 Suppl 1:67-88.

2 Morrison J, Tamang S, Mesko N, et al. Women's health groups to improve perinatal care in rural Nepal. BMC Pregnancy Childbirth 2005;5:6.

3 Prost A, Colbourn T, Seward N, et al. Women's groups practising participatory learning and action to improve maternal and newborn health in low-resource settings: a systematic review and metaanalysis. Lancet 2013;381:1736-46.

4 World Health Organization. International classification of health interventions (ICHI), n.d. Available: https://www.who.int/standards/ classifications/international-classification-of-health-interventions [Accessed 18 Mar 2021]

5 Monash University. What is the Northern Territory intervention? 2020. Available: https://www.monash.edu/law/research/centres/ castancentre/our-areas-of-work/indigenous/the-northern-territory- 
intervention/the-northern-territory-intervention-an-evaluation/whatis-the-northern-territory-intervention [Accessed 18 Mar 2021].

6 Lowell A, Kildea S, Liddle M, et al. Supporting Aboriginal knowledge and practice in health care: lessons from a qualitative evaluation of the strong women, strong babies, strong culture program. BMC Pregnancy Childbirth 2015:15:19.

7 Cyril S, Smith BJ, Possamai-Inesedy A, et al. Exploring the role of community engagement in improving the health of disadvantaged populations: a systematic review. Glob Health Action 2015;8:29842.

8 Victora CG, Barros FC. Participatory women's groups: ready for prime time? Lancet 2013;381:1693-4.

9 Atkinson J. Trauma trails, recreating song lines: the transgenerational effects of trauma in Indigenous Australia. North Melbourne: Spinifex Press, 2002.

10 Bond C, Brough M, Willis J, et al. Beyond the pipeline: a critique of the discourse surrounding the development of an Indigenous primary healthcare workforce in Australia. Aust $J$ Prim Health 2019;25:389-94.

11 Boffa J, Tilton E, Ah Chee D. Preventing alcohol-related harm in Aboriginal and Torres Strait Islander communities: the experience of an Aboriginal community controlled health service in central Australia. Aust J Gen Pract 2018;47:851-4.

12 Carlisle K, Felton-Busch C, Cadet-James Y, et al. Women's action for Mums and Bubs (womb) trial protocol: a Non-randomized stepped wedge implementation trial of participatory women's groups to improve the health of Aboriginal and Torres Strait Islander mothers and children in Australia. Front Public Health 2020;8:73.

13 Turner NN, Taylor J, Larkins S, et al. Conceptualizing the association between community participation and CQI in Aboriginal and Torres Strait Islander PHC services. Qual Health Res 2019;29:1904-15.

14 Shamseer L, Moher D, Clarke M, et al. Preferred reporting items for systematic review and meta-analysis protocols (PRISMA-P) 2015: elaboration and explanation. BMJ 2015;349:97647.

15 Preston R, Rannard S, Felton-Busch C, et al. How and why do participatory women's groups (PWGs) improve the quality of maternal and child health $(\mathrm{MCH})$ care? A systematic review protocol. BMJ Open 2019;9:e030461.

16 Taylor J, Preston R, Rannard S. How and why do participatory women's groups improve the quality of maternal and child health (MCH) care? 2019. Available: http://www.crd.york.ac.uk/PROSPERO/ display_record.php?ID=CRD42019126533 [Accessed 18 March 2021]

17 Cheng SH, Augustin C, Bethel A, et al. Using machine learning to advance synthesis and use of conservation and environmental evidence. Conserv Biol 2018;32:762-4.

18 Joanna Briggs Institute. Critical appraisal tools, 2018. Available: http://joannabriggs.org/research/critical-appraisal-tools.html [Accessed 18 June 2020].

19 Arnstein SR. A ladder of citizen participation. J Am Inst Plann 1969;35:216-24.

20 Gram L, Desai S, Prost A. Classroom, Club or collective? Three types of community-based group intervention and why they matter for health. BMJ Glob Health 2020;5:e003302.10.1136/ bmigh-2020-003302

21 Taylor J, Braunack-Mayer A, Cargo M, et al. Community and health sector partnerships for primary prevention in Australia: developing a typology. Curr Sociol 2012;60:506-21.

22 The World Bank. World bank country and lending groups, 2021. Available: https://datahelpdesk.worldbank.org/knowledgebase/ articles/906519-world-bank-country-and-lending-groups [Accessed 19 Mar 2021].

23 Earle-Crane M. The quality of prenatal care: experiences of women attending healthy baby clubs [Masters thesis. St Johns, Newfoundland: Memorial University of Newfoundland, 2000.

24 Ndirangu G, Gichangi A, Kanyuuru L, et al. Using young mothers' clubs to improve knowledge of postpartum hemorrhage and family planning in informal settlements in Nairobi, Kenya. J Community Health 2015;40:692-8.

25 Roche ML, Ambato L, Sarsoza J, et al. Mothers' groups enrich die and culture through promoting traditional Quichua foods. Matern Child Nutr 2017;13 Suppl 3:e12530.

26 O'Rourke K, Howard-Grabman L, Seoane G. Impact of community organization of women on perinatal outcomes in rural Bolivia. Rev Panam Salud Publica 1998;3:9-14.

27 Bolton M, Moore I, Ferreira A, et al. Community organizing and community health: piloting an innovative approach to community engagement applied to an early intervention project in South London. J Public Health 2016;38:115-21.

28 Dongre AR, Deshmukh PR, Garg BS. A community based approach to improve health care seeking for newborn danger signs in rural wardha, India. Indian J Pediatr 2009;76:45-50.
29 Lugo NR. Empowerment education: a case study of the resource Sisters/Compañeras program. Health Educ Q 1996;23:281-9. discussion 90-2.

30 Dickinson P, Joe T. Strengthening young mothers: a qualitative evaluation of a pilot support group program. Youth Studies Australia 2010;29:35-44.

31 Morrison J, Tumbahangphe K, Sen A, et al. Health management Committee strengthening and community mobilisation through women's groups to improve trained health worker attendance at birth in rural Nepal: a cluster randomised controlled trial. BMC Pregnancy Childbirth 2020;20:268.

32 Ronaasen J, Steenkamp L, Williams M, et al. Sakha ESETHU: nurturing value-centered group work for a community-based parent support programme in the eastern Cape, South Africa. Soc Work Groups 2021;44:364-80.

33 Colbourn T, Nambiar B, Bondo A, et al. Effects of quality improvement in health facilities and community mobilization through women's groups on maternal, neonatal and perinatal mortality in three districts of Malawi: MaiKhanda, a cluster randomized controlled effectiveness trial. Int Health 2013;5:180-95.

34 Saville N. The effect of community mobilisation through women's groups on improved IYCF: Experience from Dhanusha, Nepal. Available: http://siteresources.worldbank.org/SOUTHASIAEXT/ Resources/223546-1328913542665/UCL-MIRA_DHANUSHA.pdf [Accessed 05 Jan 2021].

35 Gram L, Skordis-Worrall J, Manandhar DS, et al. The long-term impact of community mobilisation through participatory women's groups on women's agency in the household: a follow-up study to the Makwanpur trial. PLoS One 2018;13:e0197426.

36 Manandhar DS, Osrin D, Shrestha BP, et al. Effect of a participatory intervention with women's groups on birth outcomes in Nepal: cluster-randomised controlled trial. Lancet 2004;364:970-9.

37 Skordis J, Pace N, Vera-Hernandez M, et al. Family networks and healthy behaviour: evidence from Nepal. Health Econ Policy Law 2019;14:231-48

38 Pant PR, Budhathoki B, Ellis $\mathrm{M}$, et al. The feasibility of community mobilisation for child injury prevention in rural Nepal: a programme for female community health volunteers. BMC Public Health 2015; $15: 430$

39 Morrison J, Thapa R, Hartley S, et al. Understanding how women's groups improve maternal and newborn health in Makwanpur, Nepal: a qualitative study. Int Health 2010;2:25-35.

40 Morrison J, Thapa R, Sen A, et al. Utilization and management of maternal and child health funds in rural Nepal. Community Dev $J$ 2010;45:75-89.

41 Tripathy P, Nair N, Sinha R, et al. Effect of participatory women's groups facilitated by accredited social health activists on birth outcomes in rural eastern India: a cluster-randomised controlled trial. Lancet Glob Health 2016;4:e119-28.

42 Roy SS, Mahapatra R, Rath S, et al. Improved neonatal survival after participatory learning and action with women's groups: a prospective study in rural eastern India. Bull World Health Organ 2013;91:426-33.

43 Tripathy P, Nair N, Barnett S, et al. Effect of a participatory intervention with women's groups on birth outcomes and maternal depression in Jharkhand and Orissa, India: a cluster-randomised controlled trial. Lancet 2010;375:1182-92.

44 Turan JM, Say L, Güngör AK, et al. Community participation for perinatal health in Istanbul. Health Promot Int 2003;18:25-32.

45 Wong ML, Chen PC. Self-reliance in health among village women. World Health Forum 1991;12:43-8.

46 Rath S, Nair N, Tripathy PK, et al. Explaining the impact of a women's group led community mobilisation intervention on maternal and newborn health outcomes: the Ekjut trial process evaluation. BMC Int Health Hum Rights 2010;10:25.

47 United States Agency for International Development. USAID/Burma Shaethot final performance evaluation evaluation report, 2018. Available: https://pdf.usaid.gov/pdf_docs/PA00SSDW.pdf [Accessed 18 Mar 2021].

48 Ministry of Health, UNICEF Sri Lanka. Review of the functioning and impact of mother support groups in the Northern and eastern provinces of Sri Lanka, 2015. Available: https://www.unicef.org/ srilanka/media/336/file/MOTHER\%20SUPPORT\%20GROUPS.pdf [Accessed 18 Mar 2021].

49 Gill K. If we walk together - communities, NGOs, and Government in partnership for health : the Hyderabad experience, 1999. Available: http://documents.worldbank.org/curated/en/383321468772798321/ If-we-walk-together-communities-NGOs-and-Government-inpartnership-for-health-the-Hyderabad-experience [Accessed $19 \mathrm{Mar}$ 2021]. 
50 Kruske S, Schmied V, Sutton I, et al. Mothers' experiences of facilitated peer support groups and individual child health nursing support: a comparative evaluation. J Perinat Educ 2004;13:31-8.

51 Quigley P, Green C, Soyoola M, et al. Empowering women and communities to promote universal health coverage in rural Zambia. Dev Pract 2018;28:1094-100.

52 Damtew ZA, Karim AM, Chekagn CT, et al. Correlates of the women's development army strategy implementation strength with household reproductive, maternal, newborn and child healthcare practices: a cross-sectional study in four regions of Ethiopia. BMC Pregnancy Childbirth 2018;18:373.

53 Nishiuchi M. Mothers in community health care activities--The Suita Mother's Club. JOICFP Rev 1985:5-16.

54 Morrison J, Osrin D, Alcock G, et al. Exploring the equity impact of a maternal and newborn health intervention: a qualitative study of participatory women's groups in rural South Asia and Africa. Int J Equity Health 2019;18:55.

55 Sharma S, Mehra D, Akhtar F, et al. Evaluation of a community-based intervention for health and economic empowerment of marginalized women in India. BMC Public Health 2020;20:1766.

56 Cohen JM, Uphoff NT. Participation's place in rural development: Seeking clarity through specificity. World Dev 1980;8:213-35.

57 Byass P. The potential of community engagement to improve mother and child health in Ethiopia - what works and how should it be measured? BMC Pregnancy Childbirth 2018;18:366.

58 Abimbola S. Beyond positive a priori bias: reframing community engagement in LMICs. Health Promot Int 2020;35:598-609.

59 George AS, LeFevre AE, Schleiff M, et al. Hubris, humility and humanity: expanding evidence approaches for improving and sustaining community health programmes. BMJ Glob Health 2018;3:e000811.

60 Haldane V, Chuah FLH, Srivastava A, et al. Community participation in health services development, implementation, and evaluation: a systematic review of empowerment, health, community, and process outcomes. PLoS One 2019;14:e0216112.
61 Brunton G, Thomas J, O'Mara-Eves A, et al. Narratives of community engagement: a systematic review-derived conceptual framework for public health interventions. BMC Public Health 2017;17:944.

62 Preston $\mathrm{R}$, Waugh $\mathrm{H}$, Larkins $\mathrm{S}$, et al. Community participation in rural primary health care: intervention or approach? Aust J Prim Health 2010;16:4-16.

63 Hoon Chuah FL, Srivastava A, Singh SR, et al. Community participation in general health initiatives in high and uppermiddle income countries: a systematic review exploring the nature of participation, use of theories, contextual drivers and power relations in community participation. Soc Sci Med 2018;213:106-22.

64 Mannell J, Davis K. Evaluating complex health interventions with randomized controlled trials: how do we improve the use of qualitative methods? Qual Health Res 2019;29:623-31.

65 Palmer VJ, Weavell W, Callander R, et al. The participatory Zeitgeist: an explanatory theoretical model of change in an era of coproduction and codesign in healthcare improvement. Med Humanit 2019;45:247-57.

66 Paul VK. Participatory women's groups: time for integration into programmes. Lancet Glob Health 2016;4:e74-5.

67 Woelk GB. Cultural and structural influences in the creation of and participation in community health programmes. Soc Sci Med 1992;35:419-24.

68 Olivier de Sardan J-P, Diarra A, Moha M. Travelling models and the challenge of pragmatic contexts and practical norms: the case of maternal health. Health Res Policy Syst 2017;15:60.

69 Sondaal AEC, Tumbahangphe KM, Neupane R, et al. Sustainability of community-based women's groups: reflections from a participatory intervention for newborn and maternal health in Nepal. Community Dev J 2019;54:731-49.

70 Moher D, Liberati A, Tetzlaff J, et al. Preferred reporting items for systematic reviews and meta-analyses: the PRISMA statement. PLoS Med 2009;6:e1000097. 